\title{
Short Communication: Contribution of Vibration and Noise During Milking to the Somatic Cell Count of Milk
}

\author{
L. Gygax ${ }^{\star 1}$ and D. Nosal† \\ ${ }^{*}$ Centre for Proper Housing of Ruminants and Pigs, Swiss Federal Veterinary Office, and \\ †Construction \& Animals, Agroscope FAT Tänikon, Ettenhausen 8356, Switzerland
}

\begin{abstract}
We investigated the hypothesis that somatic cell counts (SCC) in milk are influenced by the vibration and noise experienced by dairy cows during milking. We therefore measured vibration and noise on 50 Swiss dairy farms (with herringbone, autotandem, side-byside, or carousel parlors), where we also collected bulk tank SCC. Somatic cell counts increased with an increasing intensity of vibration but not with acoustic noise. Cows milked in autotandem and side-by-side parlors had lower SCC than those in the other 2 types of milking parlors. On 12 farms where the milking system was modified to reduce vibration and noise, SCC also dropped. In addition, the relative improvement in SCC seemed to be correlated with the relative improvement in the reduction of vibration but not with the improvement in acoustic noise. A reduction in vibration (structure-borne sonic waves) seemed to improve udder health, which may have been mediated by the increased well-being and reduced stress of cows during milking. Key words: dairy cow, somatic cell count, vibration, noise
\end{abstract}

Beef cattle react with unrest and an increased heart rate to noise during handling, which has been interpreted as a reaction of fearfulness (Waynert et al., 1999). Head et al. (1993) have also suggested that noise might influence the well-being of dairy cows and thus, indirectly, their health and milk production. In a previous study, this notion could not be supported when dairy cows were subjected to aircraft noise just before the onset of milking (Head et al., 1993). Dairy cows are faced much more directly with sonic waves (noise and vibration) in the milking parlor, and we investigated whether and to what extent structure-borne sonic waves (vibration) and airborne sonic waves (noise) in the milking parlor coincide with increased SCC, one likely indicator of udder health (Harmon, 1994).

Received September 16, 2005.

Accepted February 15, 2006.

${ }^{1}$ Corresponding author: lorenz.gygax@fat.admin.ch
Measurements were conducted on 50 Swiss dairy farms (29 herringbone, 11 autotandem, 9 side-by-side, and 1 carousel milking parlor; Nosal et al., 2004) representing the common types of milking parlors in Switzerland. On each farm, vibration $\left(\mathrm{m} / \mathrm{s}^{2}\right.$, at the manure splash guards; integrating vibration meter, model 2513, Brüel \& Kjær, Nærum, Denmark; 0.1 to $100 \mathrm{~m} / \mathrm{s}^{2}$ ) and noise $(\mathrm{dB}$, at approximately $1.2 \mathrm{~m}$ height in the milking stall head area; sound level meter, model 2232, Brüel \& Kjær; 34 to $130 \mathrm{~dB}$ ) were measured while the milking machine was running. The highest amplitudes of vibration and noise were noted because these extremes were thought to have an effect on the well-being of the cows in the milking parlor.

We measured SCC on farms using bulk tank milk samples. Such samples have been shown to be relatively independent of management factors (because only 18\% of the variability could be explained in a model by van Schaik et al., 2005) but to be correlated with a weighted average individual SCC (Valde et al., 2005). To ensure a permanently high level of milk quality, all farms in Switzerland are subject to monthly milk SCC checks, for which a sample is taken from the storage tank (thus, an average SCC weighted by the individual milk yield is measured per herd). All the SCC measurements for the year preceding the vibration and noise measurements were collected and averaged for each farm in the study. On 12 of the farms (9 herringbone, 2 autotandem, and 1 side-by-side), the milking parlor was then modified to reduce vibration and noise levels during milking. Modifications were made specifically on each farm and included putting the vacuum pump on rubber supports, using rubber tubing to connect the vacuum pump to the exhaust and the main conduit, installing regulatory buffer tanks between the pump and the conduits, installing buffer tanks between the main conduit and the pulsator, using absorbing ducts to install all tubes and tanks, and connecting the pulsators with elastic and dampening tubes. The extent of the reduction was measured, and SCC data were again collected for at least 3 mo directly following the modification and were averaged per farm.

A generalized least squares approach was used in the software R (R Development Core Team, 2005) to model 
$y=\alpha+\beta_{1} \cdot X_{1}+\beta_{2} \cdot X_{2}+E^{\mathrm{TP}}+\beta_{3} \cdot \mathrm{X}_{1} \cdot X_{2}$, where $y$ is the logarithm-transformed SCC, $\alpha$ is the intercept, $X_{1}$ is the vibration (continuous), $X_{2}$ is noise (continuous), $E^{\mathrm{TP}}$ is the type of milking parlor (factor using treatment contrasts with the most common herringbone type as the level with which the other types were compared), and $X_{1} \cdot X_{2}$ is the interaction between vibration and noise. Estimates of the parameters $\alpha$ and $\beta$ were based on restricted maximum likelihood (Pinheiro and Bates, 2000; Pinheiro et al., 2005). The interaction of vibration and noise did not reach significance $\left(F_{1,43}=0.23\right.$, $P=0.63$ ) and was an order of magnitude smaller than the of vibration. Including the interaction effect did not influence the effect of vibration, but the effect of the parameter "noise" was even smaller in the model with the interaction. Thus, the interaction was omitted from the model presented above. The interactions between type of milking parlor and vibration or noise were not included because of the size of the data set. In addition, the heteroscedasticity arising in the different types of milking parlors was accounted for.

The SCC of the 12 farms after modification were compared with their values before modification using the Wilcoxon test. In addition, we investigated whether a greater reduction in vibration and noise led to a greater reduction in SCC using a generalized least squares approach analogous to the one just described. Because the types of milking parlors that were modified mainly consisted of herringbone systems, the variable "type of milking parlor" was not included as a fixed effect in this model but still remained as a term to account for differences in variability. We checked that the 2 autotandem parlors and the 1 side-by-side parlor in the sample did not stand out on a scatter plot (Figure 1c). For the effects of modification, the response and explanatory variables were investigated in their raw form, log-transformed, and expressed as relative changes in comparison with the state before modification. Only one model, based on the relative change of all these variables, is presented here.

To enable us to compare the relative importance of vibration and noise directly, that is, to compare their estimated slope parameters, these variables were normalized (i.e., from each value the mean was subtracted and this difference was divided by the standard deviation). To satisfy statistical assumptions, SCC were logtransformed in the model of the full data set. Assumptions of the models were checked using a graphical analysis of residuals.

There was no correlation between measures of vibration and noise (full data set: Kendall $\tau=0.14, P=0.16$, $\mathrm{n}=50$; modified parlors: Kendall $\tau=0.05, P=0.83, \mathrm{n}=$ 12 ); thus, the influences of vibration and noise could be estimated independently in this data set.
In the analysis of the full data set, SCC values increased perceptibly with increasing vibration $\left(\mathrm{F}_{1,44}=\right.$ 33.76, $P<0.001$; Figure 1a), whereas the relationship with noise did not reach significance $\left(\mathrm{F}_{1,44}=0.39, P=\right.$ $0.53)$. The slope parameter was much greater for vibration than for noise (by a factor of approximately 90 on the log scale). The type of milking parlor had a small influence on SCC, with SCC values lower in the autotandem and side-by-side parlors and somewhat higher in the (one) carousel parlor compared with the herringbone parlors $\left(\mathrm{F}_{3,44}=3.57, P=0.021\right)$.

The modification led to a median decrease in vibration and noise of $0.35 \mathrm{~m} / \mathrm{s}^{2}$ and $6 \mathrm{~dB}$, respectively (range: 0 to $0.9 \mathrm{~m} / \mathrm{s}^{2}, 3$ to $24 \mathrm{~dB}$ ). This absolute decrease was highly correlated with the relative decrease (Pearson $r=0.80$ for vibration and $r=0.99$ for noise). The modification in the milking parlors led to a decrease in SCC on all farms (Wilcoxon test: $\mathrm{V}=78, P<0.001$; Figure 1b). Also, the relative improvement in SCC increased with the relative reduction of vibration $\left(\mathrm{F}_{1,9}=13.74\right.$, $P=0.005$; Figure 1c), but not with the relative reduction of noise $\left(\mathrm{F}_{1,9}=0.02, P=0.89\right)$. Again, the estimated slope parameter for vibration was much greater than for noise (about 20-fold). As shown in Figure 1c, this relationship mainly depended on one farm (where vibration was at a low level even prior to the modification), because the relative reduction of vibration on the other farms was within a rather narrow range and, accordingly, these farms had similar relative improvements in SCC. If this farm was omitted from the analysis, then the slope was still positive but much weaker (Figure $1 \mathrm{c} ; \mathrm{F}_{1,8}=0.42, P=0.53$ ). Even when considering all the data, no single points obviously deviated from the suggested relationship between the relative reduction of vibration and relative improvement in SCC.

In a previous study, Head et al. (1993) found no influence of noise on behavioral parameters reflecting wellbeing or on production (milk yield and milk composition) in dairy cows faced with aircraft noise several times a day independently of milking times. In our study, we found a clear interrelationship between high SCC values and vibration. This seeming difference in comparison with the results of Head et al. (1993) may be due either to the simultaneous exposure to vibration and milking in our study or to the extension of our investigation to structure-borne sonic waves (vibration) in addition to acoustic noise. It may be that the negative effect of vibration on SCC is mediated by reduced oxytocin levels under stressful conditions, leading to a reduced milk yield. This would in turn increase residual milk, which could result in subclinical mastitis and increased SCC (Bruckmaier and Blum, 1998; Stelwagen et al., 2000; Rushen et al., 2001). Because the modification was likely to lead to a more stable vacuum at 
a

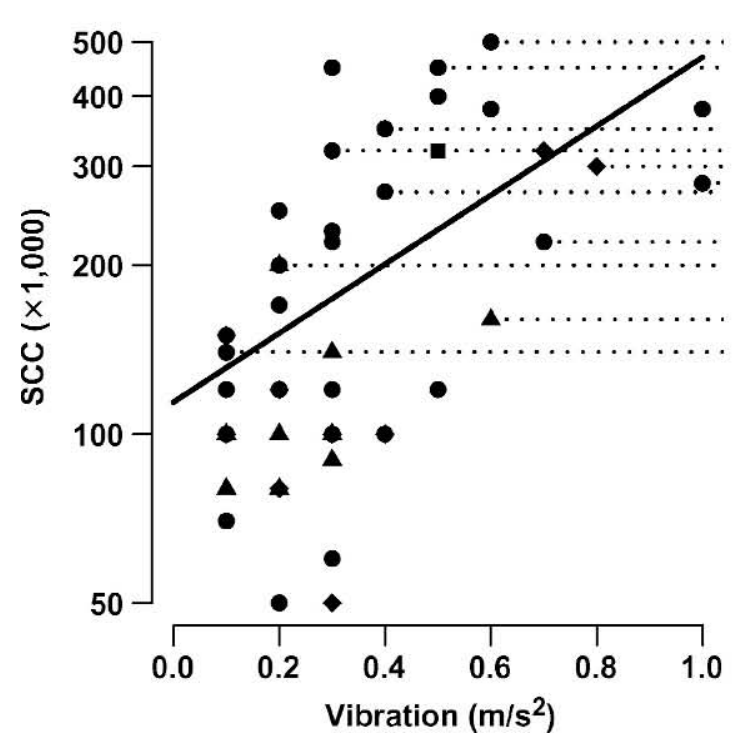

b

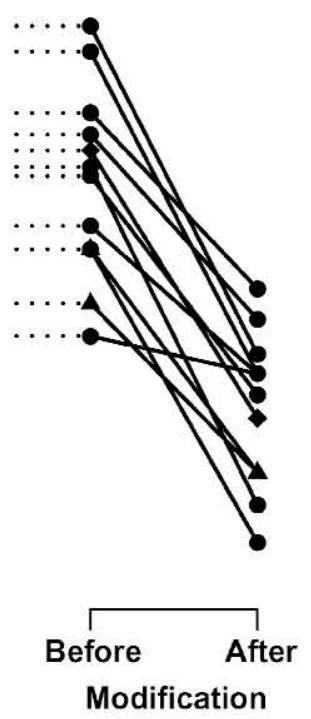

C

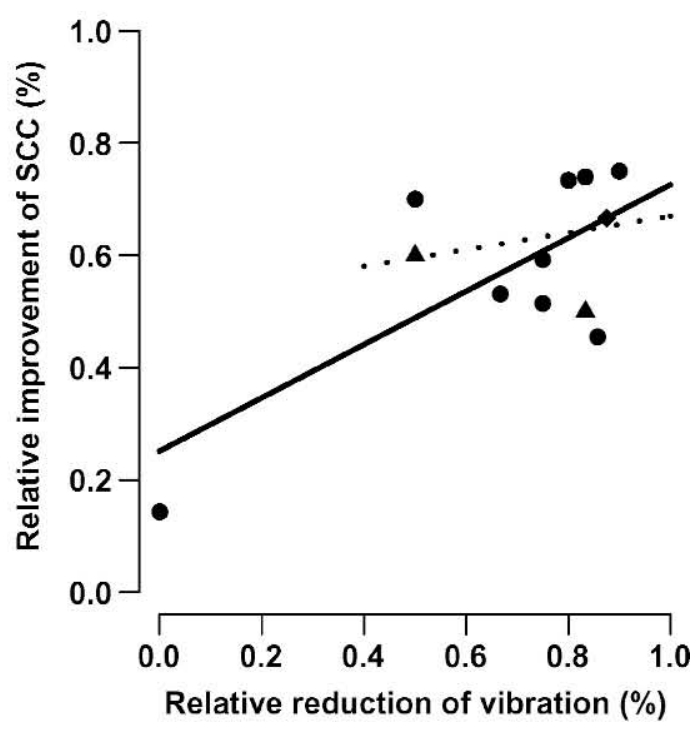

Figure 1. a) Interrelation between SCC and vibration (fitted line is given for the median of the noise measurements at $73 \mathrm{~dB}$ and for the most common type of milking parlor, the herringbone); b) Reduction in SCC after modification to reduce vibration and noise; c) Interrelation between the relative improvement of SCC and the relative reduction of vibration (fitted lines are given for the median of relative noise improvement of 34\%; solid line: model with all data points; dotted line: model omitting the one farm where vibration was not reduced). Symbols reflect the type of milking parlor: herringbone $(\bullet)$, autotandem $(\mathbf{\Delta})$, side-by-side $(\bullet)$, and carousel ( $\mathbf{\square})$.

the teats on some farms, part of the effect might have been due to direct physical impact on the teats. In addition, we cannot completely rule out the possibility that the strong vibrations observed during milking in this study were an indication of a more general lack of careful management and that the high SCC values were a result of the same negligent management practices rather than a direct consequence of the vibrations. On the other hand, it is unlikely that only farmers who were well aware of their management practices were participating in the modification subset, because vibrations are not easily perceived by humans. Thus, even farmers with careful management practices may not have been aware of a vibration problem. In addition, we found that the SCC dropped on all the farms that had modified their milking systems. It is unlikely that such an investment in construction resulted in a general change in management practices, although this cannot be excluded on the basis of our design. Finally, there are known indications that stressful events may increase SCC in dairy cows (Harmon, 1994).

To explain the remaining variability in SCC even after controlling for vibration, as shown in Figure 1a, SCC should be collected for the individual cows, and more detailed characteristics of the cows and the farms need to be recorded in future studies. These characteristics could include the level of subclinical and clinical mastitis, parity, day into lactation, milk yield, breed, illnesses, milking technique (e.g., strength of the vacuum), space allowance, herd size, feeding regimen, season, and management intensity. In addition to a maximum value of noise and vibration, integrated measures of extent and duration might be valuable for inferring stress levels. This would help to unravel the complicated web of causal relationships that result in increased SCC.

\section{ACKNOWLEDGMENTS}

We would like to thank R. Rutishauser for data handling; E. Bilgery and A. Oertle for their technical expertise and support; E. Hillmann, B. Wechsler, and 2 anonymous reviewers for their critical comments; and the Swiss Federal Veterinary Office (Berne, Switzerland) for financial support to L. Gygax (grant no. 2.03.05).

\section{REFERENCES}

Bruckmaier, R. M., and J. W. Blum. 1998. Oxytocin release and milk removal in ruminants. J. Dairy Sci. 81:939-949.

Harmon, R. J. 1994. Physiology of mastitis and factors affecting somatic cell counts. J. Dairy Sci. 77:2103-2112.

Head, H. H., R. C. Kull, M. S. Campos, K. C. Bachman, C. J. Wilcox, L. L. Cline, and M. J. Hayen. 1993. Milk yield, milk composition, and behavior of Holstein cows in response to jet aircraft noise before milking. J. Dairy Sci. 76:1558-1567. 
Nosal, D., R. Rutishauser, E. Bilgery, and A. Oertle. 2004. Lärm und Vibration als Stressfaktoren beim Melken. FAT-Ber. 625:1-12. http://www.fat.admin.ch Accessed May 10, 2006.

Pinheiro, J. C., and D. M. Bates. 2000. Mixed-Effects Models in S and S-Plus. Springer, New York, NY.

Pinheiro, J. C., D. M. Bates, S. DebRoy, and D. Sarkar. 2005. NLME: Linear and Nonlinear Mixed Effects Models. R Package Version 3.1-60. http://www.R-project.org

$\mathrm{R}$ Development Core Team. 2005. R: A language and environment for statistical computing. R Foundation for Statistical Computing, Vienna, Austria. http://www.R-project.org

Rushen, J., L. Munksgaard, P. G. Marnet, and A. M. de Passillé. 2001. Human contact and the effects of acute stress on cows at milking. Appl. Anim. Behav. Sci. 73:1-14.
Stelwagen, K., H. Hopster, J. T. N. van der Werf, and H. J. Blokhuis. 2000. Effects of isolation stress on mammary tight junctions in lactating dairy cows. J. Dairy Sci. 83:48-51.

Valde, J. P., O. Osteras, and E. Simensen. 2005. Description of herd level criteria for good and poor udder health in Norwegian dairy cows. J. Dairy Sci. 88:86-92.

van Schaik, G., L. E. Green, D. Guzman, H. Esparza, and N. Tadich. 2005. Risk factors for bulk milk somatic cell counts and total bacterial counts in smallholder dairy farms in the 10th region of Chile. Prev. Vet. Med. 67:1-17.

Waynert, D. F., J. M. Stookey, K. S. Schwartzkopf-Genswein, J. M. Watts, and C. S. Waltz. 1999. The response of beef cattle to noise during handling. Appl. Anim. Behav. Sci. 62:27-42. 